\title{
Throughput maximization in discrete rate based full duplex wireless powered communication networks
}

\author{
Muhammad Shahid Iqbal $^{1} \odot \mid$ Yalcin Sadi ${ }^{2}$ | Sinem Coleri ${ }^{1}$
}

\author{
${ }^{1}$ Department of Electrical and Electronics \\ Engineering, Koc University, Istanbul, \\ Turkey \\ ${ }^{2}$ Department of Electrical and Electronics \\ Engineering, Kadir Has University, \\ Istanbul, Turkey

\section{Correspondence} \\ Sinem Coleri, Department of Electrical \\ and Electronics Engineering, Koc \\ University, Istanbul 34450, Turkey. \\ Email: scoleri@ku.edu.tr

\section{Funding information} \\ Scientific and Technological Research \\ Council of Turkey, Grant/Award Number: \\ $117 \mathrm{E} 241$.
}

\begin{abstract}
In this study, we consider a discrete rate full-duplex wireless powered communication network. We characterize a novel optimization framework for sum throughput maximization to determine the rate adaptation and transmission schedule subject to energy causality and user transmit power. We first formulate the problem as a mixed integer nonlinear programming problem, which is hard to solve for a global optimum in polynomial-time. Then, we investigate the characteristics of the solution and propose a polynomial time heuristic algorithm for rate adaptation and scheduling problem. Through numerical analysis, we illustrate that the proposed scheduling algorithm outperforms the conventional schemes such as equal time allocation half-duplex and on-off transmission schemes for different initial battery levels, hybrid access point transmit power and network densities.
\end{abstract}

\section{K E Y W O R D S}

rate adaptation, scheduling, throughput maximization, wireless powered communication networks

\section{I INTRODUCTION}

For many wireless sensor applications, wireless powered communication network (WPCN) is preferred since it is easy to install, low cost and flexible. In WPCN, a dedicated hybrid access point (HAP) transmits energy in the downlink for the users, that is, sensors or machine type communication devices, and the users harvest this energy for their uplink data transmission. Some studies ${ }^{1,2,3}$ focus on the throughput maximization considering half-duplex models, in which energy and information are transferred in sequential non-overlapping slots. This half-duplex scheme results in equal energy harvesting (EH) duration for all the users. Therefore, the transmission order does not have any effect on the throughput of the users. Due to the recent advances in self-interference cancellation (SIC) techniques ${ }^{4}$ and their practical implementations, ${ }^{5}$ full-duplex (FD) technique is being investigated for WPCN in which users can perform simultaneous energy harvesting and data transmission. For the FD model, ${ }^{6,7}$ present the sum throughput maximization in which either only HAP or users and HAP both are operating in FD mode. In the FD mode, users can harvest energy during their own and previously scheduled users transmission, which results in an uneven energy harvesting time for each user, hence, making scheduling critical. The authors in References 8, 9, 10, 11 pay attention to scheduling but in a limited context: In Reference 8 authors use a classic combinatorial optimization algorithm known as Hungarian algorithm to schedule the users, which requires exponential computational complexity for such sequence dependent networks. Reference 9 divides the whole frame into a fixed number of time slots of equal length, and then allocates them to the users, which results in the under-utilization of the resources. On the other hand, ${ }^{10}$ considers a continuous transmission rate model to maximize the sum throughput and ${ }^{11}$ considers beam-forming and scheduling to minimize the data queuing delay of the users in a multi-carrier heterogeneous network. In our previous studies, ${ }^{12,13,14}$, we propose minimum length scheduling

This work is supported by Scientific and Technological Research Council of Turkey Grant \#117E241.

[Correction added on 14 September 2020, after first online publication: the layout has been amended to fit in 6 pages.] 


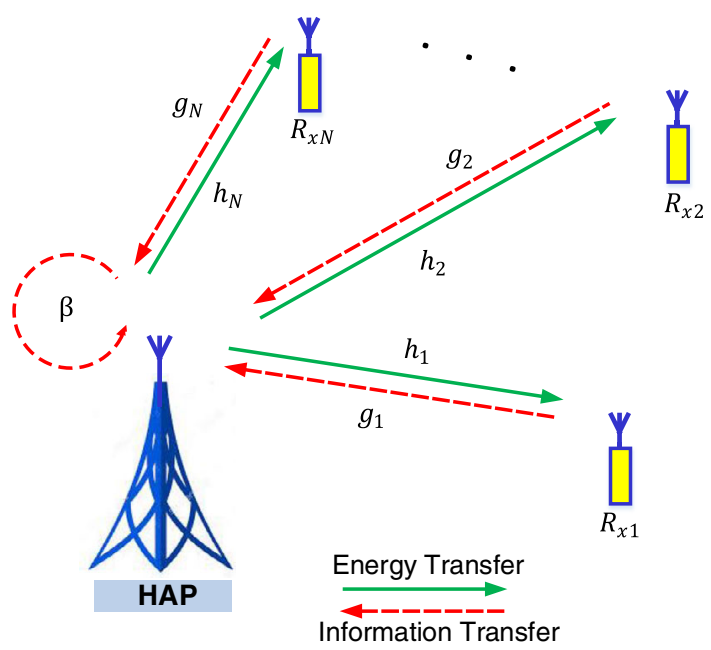

F I G U R E 1 Architecture of wireless powered communication network

(MLS) for continuous, constant and discrete rate based WPCN. Discrete rate model is more realistic than the continuous rate model since a wireless transmitter can only work with a limited rate levels however, for WPCN, only ${ }^{14}$ considers a discrete rate model for MLS problem. The goal of this letter is to analyze a more practical discrete rate based in-band full-duplex WPCN with the objective of sum throughput maximization (STM) subject to the user maximum transmit power, and energy causality constraint considering the initial battery levels.

\section{2 | SYSTEM MODEL}

The system model and assumptions are described as follows:

The full-duplex WPCN architecture depicted in Figure 1, consists of a HAP and a set of users $\mathcal{N}=\{1,2, \cdots, N\}$. The uplink and downlink channel gains of user $i$ are denoted by $g_{i}$ and $h_{i}$, respectively. The channel gains remain the same within a transmission block but may change in the other blocks, that is, block fading. The HAP has a stable power connection and continuously radiates power $P_{h}$. The users store the harvested energy in a battery with initial battery level $B_{i}$ at the start of the frame. The users transmit their data to the HAP by using time division multiple access protocol. The energy harvesting rate of user $i$ is $C_{i}=\eta_{i} h_{i} P_{h}$, where, $\eta_{i}$ is the antenna efficiency. We use a practical discrete rate transmission model, in which a finite set of $M$ transmission rates $\mathbf{R}=\left(r^{1}, r^{2}, \cdots, r^{M}\right)$ and a finite set of $M$ SINR levels $\gamma=\left(\gamma^{1}, \gamma^{2}, \cdots, \gamma^{M}\right)$ are determined such that user $i$ can transmit at rate $r^{k}$ successfully in the allocated time slot if the achieved SINR for user $i$ satisfies:

$$
\gamma_{i}=\frac{P_{i} g_{i}}{\sigma_{o}^{2}+\beta P_{h}} \geq \gamma^{k},
$$

where the term $\beta P_{h}$ is the power of self-interference at the HAP and $\sigma_{o}^{2}$ is the noise power.

\section{3 | SUM THROUGHPUT MAXIMIZATION PROBLEM}

In this section, we introduce the discrete rate based sum throughput maximization problem, referred as DR-STMP.

DR-STMP:

maximize

$$
\sum_{i=1}^{N} \tau_{i} \chi_{i}
$$

subject to

$$
\begin{gathered}
P_{i} g_{i}-\left(\sum_{k=1}^{M} z_{i k} \gamma^{k}\right)\left(\sigma_{o}^{2}+\beta P_{h}\right) \geq 0, \quad i \in\{1,2, \cdots, N\} \\
B_{i}+C_{i} \tau_{0}+C_{i} \sum_{j=1, j \neq i}^{N} a_{i j} \tau_{j}+C_{i} \tau_{i}-P_{i} \tau_{i} \geq 0, \quad \forall i \in\{1,2, \cdots, N\}
\end{gathered}
$$




$$
\begin{gathered}
P_{i} \leq P_{\text {max }}, \quad \forall i \in\{1,2, \cdots, N\} \\
\chi_{i}=\sum_{k=1}^{M} z_{i k} r^{k}, \quad \forall i \in\{1,2, \cdots, N\} \\
\sum_{k=1}^{M} z_{i k}=1, \quad \forall i \in\{1,2, \cdots, N\} \\
a_{i j}+a_{j i}=1, i \neq j, \quad \forall i, j \in\{1,2, \cdots, N\} \\
\sum_{i=0}^{N} \tau_{i}=T,
\end{gathered}
$$

where the variables of the problem are $P_{i}$, the transmit power of user $i$, for $i \in\{1,2, \cdots, N\}$; $\tau_{i}$, the transmission time of user $i$, for $i \in\{1,2, \cdots, N\} ; a_{i j}$, a binary variable which takes value 1 if user $i$ is scheduled before user $j$ and 0 otherwise, for $i, j \in\{1,2, \cdots, N\}$; and $z_{i k}$, a binary variable which takes value 1 if user $i$ is allocated to rate $r^{k}$ and 0 otherwise, for $i \in\{1,2, \cdots, N\}, k \in\{1,2, \cdots$, $M$ \}. In addition, $\tau_{0}$ denotes an initial waiting time duration during which all users only harvest energy without transmitting any information and $P_{\max }$ is the maximum allowed transmit power for any user. The objective is to maximize the sum throughput as given by Equation (2a). Equation (2b) represents the constraint on satisfying the rate adaptation of the users. Equation (2c) gives the energy causality constraint, that is, consumed energy should be less than the available energy. Equation (2d) represents the maximum transmit power constraint for the users to avoid interference to the nearby users. Equation (2e) and Equation (2f) together represent the selection of one of the available transmission rates as the transmission rate of user $i$. Equation (2g) is a scheduling constraint, that is, if user $i$ is transmitting before user $j$ then user $j$ cannot transmit before user $i$. Finally, Equation ( $2 \mathrm{~h}$ ) is the frame length constraint, where the length of the frame is denoted by $T$. The optimization problem formulation presented in Equation (2) is a mixed integer non-linear programming (MINLP) problem, which is generally hard to solve for a global optimum in polynomial-time.

\section{4 | OPTIMALITY ANALYSIS}

We start by investigating the problem for user $i$. Every user requires a certain power to achieve a particular transmission rate. Let $P_{i}^{k}$ be the minimum required transmission power to achieve the $k^{\text {th }}$ transmission rate via satisfying the SNR constraint $\gamma_{i} \geq \gamma^{k}$ using the harvested energy. Then, constraint $2 \mathrm{~b}$ should hold with equality and the required transmission power is given by:

$$
P_{i}^{k}=\frac{\gamma^{k}\left(\sigma_{0}^{2}+\beta P_{h}\right)}{g_{i}}, \quad \forall k \in\{1,2, \cdots, M\}
$$

The $P_{\max }$ constraint in Equation 2d needs to be satisfied: if $P_{i}^{k}>P_{\max }$, then the $k^{\text {th }}$ transmission rate is infeasible for the particular user. This limitation implies that every user may afford a different set of rate levels. Let $\mathbf{r}_{i}$ denote the set of rates user i can afford, where $\mathbf{r}_{i} \subseteq \mathbf{R}$ and $\mathbf{n}_{i}$ is the set of users which have rate level $i$, i.e., $r^{i}$ as their highest affordable rate level at the scheduling decision time where $\mathbf{n}_{i} \subseteq \mathcal{N}$.

Lemma 1. In the optimal solution of STMP, for any two users $i$ and $j$ transmitting at $P_{\max }$ such that $\max \mathbf{r}_{i}>\max \mathbf{r}_{j}$, if $\tau_{i}=0$, then $\tau_{j}=0$.

Proof. Suppose that $\tau^{*}=\left[\tau_{1}^{*}, \tau_{2}^{*}, \ldots, \tau_{N}^{*}\right]$ is the optimal transmission time for an $\mathcal{N}$ such that $\tau_{i}^{*}=0$ and $\tau_{j}^{*}>0$ for some $i$ and $j$ such that $\max \mathbf{r}_{i}>\max \mathbf{r}_{j}$. For some $\tau$ ' $>0$, without violating energy causality of user $i$, transmission time of user $j$ can be divided into two time slots $\tau_{j}^{*}-\tau^{\prime}$ and $\tau$, each assigned to users $j$ and $i$, respectively. Then, the sum throughput is increased by $\tau^{\prime}\left(\max \mathbf{r}_{i}-\max \mathbf{r}_{j}\right)$ which is strictly positive. This is a contradiction.

Lemma 1 suggests that high rate users should be prioritized for STM, an optimal schedule may not contain all users as long as the maximum throughput is achieved by a subset of users. Depending on the energy available for users; some high rate users may utilize the entire scheduling frame. For instance, if a user can feasibly afford maximum transmission rate for the entire frame, the optimal schedule will only contain this particular user as indicated by the following corollary of Lemma 1.

Corollary 1. Let user $i$ can afford maximum transmission rate for the entire frame then, there exists an optimal solution in which $\tau_{i}=T$; i.e., user $i$ is allocated to the entire scheduling frame. 
Now, let $t_{s_{i}}^{k}$ and $t_{e_{i}}^{k}$ be the times user $i$ can afford data transmission using rate level $k$ while allocated at the start and end of the frame respectively. These transmission times can be evaluated as $t_{s_{i}}^{k}=B_{i} / P_{i}^{k}$ and $t_{e_{i}}^{k}=\left(B_{i}+C_{i} T\right) / P_{i}^{k}$. Since the user can afford $k^{t h}$ rate for a longer time if it is allocated at the end of the frame due to more energy harvesting time. This suggests that throughput can be increased if the transmission of user with higher energy harvesting capability is delayed. Let $\Delta_{i}^{k}$ be the difference between $t_{e_{i}}^{k}$ and $t_{s_{i}}^{k}$, i.e., $\Delta_{i}^{k}=t_{e_{i}}^{k}-t_{s_{i}}^{k}$ for rate level $k$ then among all the users having rate level $k$ as their highest feasible rate level, it is better to allocate a user with higher $\Delta_{i}^{k}$ at the end of the frame as compared to a user with smaller $\Delta_{i}^{k}$. This suggest that the higher $\Delta_{i}^{k}$ users should be prioritized by the scheduling algorithm, that is, they should be allocated at the end of the frame. Based on this analysis and Lemma 1, we propose a polynomial time heuristic scheduling algorithm which maximizes the sum throughput presented in Algorithm 1.

\section{5 | SCHEDULING ALGORITHM}

Based on the analysis of the joint rate adaptation and scheduling problem, we present the maximum rate first algorithm (MRFA), as given in Algorithm 1. The algorithm aims to pick the transmission order which maximizes the sum throughput. As suggested by the optimality analysis, MRFA starts by allocating the user that can afford maximum possible rate for maximum time and iteratively determines the schedule. Note that the algorithm starts allocation from the end of the frame so that the user with higher energy harvesting rate can have more time to harvest the energy. The algorithm is described as follows. The input of MRFA is a set of users, denoted by $\mathcal{N}$ with characteristics specified in Section 2 and the sets of users with $k^{\text {th }}$ rate level as their maximum feasible rate level denoted by $\mathbf{n}_{k} \forall k \in\{1,2, \cdots, M\}$ (line 1). The Algorithm is initialized by $k=M$, i.e., the highest rate level. MRFA starts by checking whether $\mathbf{n}_{k}$ is an empty set, which means no user can afford the $k^{\text {th }}$ rate level, therefore, it will lower down the rate level until it finds a feasible rate level (lines 5-7). Once the feasible rate level is determined, MRFA evaluates the time difference $\Delta$ for all the users in set $\mathbf{n}_{k}$ (lines 8-13). After the evaluation of the transmission power and time difference, for best utilization of transmission time, the algorithm allocates the transmission time to a user with the best energy harvesting capability and can improve the throughput most. Therefore, the algorithm starts allocation with the highest $\Delta$ for this rate level and allocates the maximum feasible transmission time to this user without violating energy causality constraint (lines 14-15). Then, the frame length (line 16), schedule (line 17) and set $\mathbf{n}_{k}$ (line 18) are updated accordingly. Then, the algorithm is re-executed for the remaining users (lines 4-19). If the unallocated time duration is 0 at any step, MRFA terminates by not scheduling the remaining users (lines 20-22) otherwise it schedules all the users. Upon termination, MRFA outputs the transmission times $\tau((\mathcal{S})$ ), transmission schedule $\mathcal{S}$, corresponding rates $\chi(\mathcal{S})$, and sum throughput $\Re(\mathcal{S})$. The computational complexity of MRFA is $\mathcal{O}\left(N^{2}\right)$ for $N$ users. The algorithm evaluates the transmission time, power and rate for one user in each iteration, and then determines the transmission rate, followed by evaluating the time difference $\Delta$ for each user. Assuming $N>M$, the complexity of each iteration is $\mathcal{O}(N)$ with total number of $\mathrm{N}$ iterations for $\mathrm{N}$ users. Algorithm 1 gives the algorithm which maximizes the sum throughput.

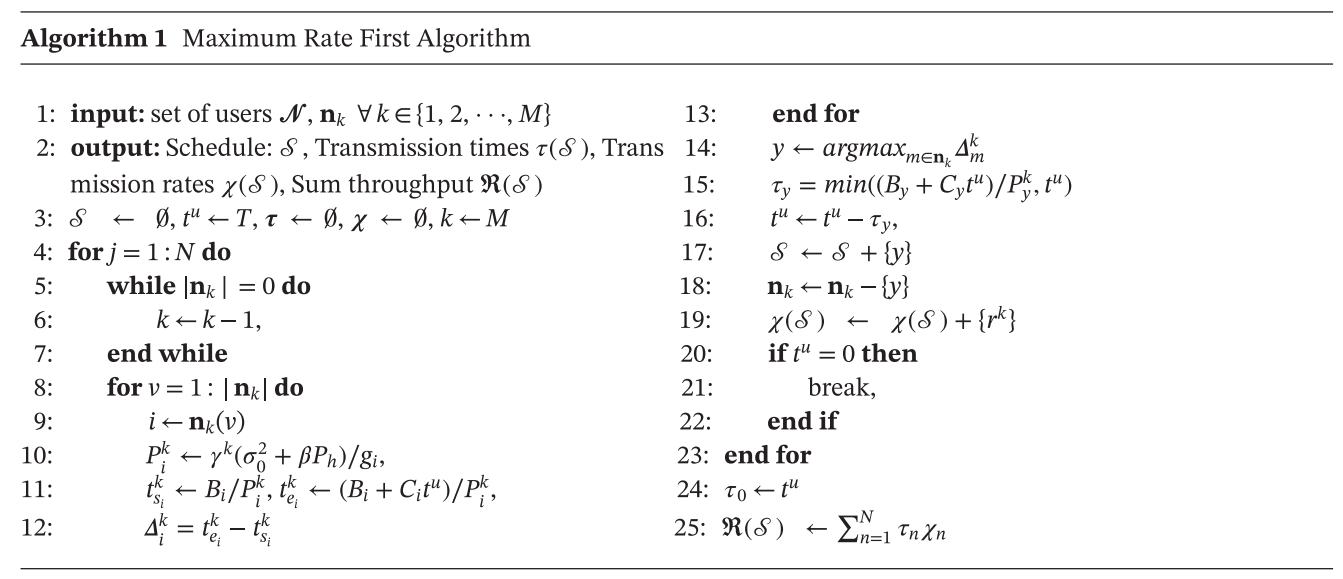

\section{6 | PERFORMANCE EVALUATION}

The goal of this section is to evaluate the performance of the proposed heuristic algorithm MRFA in comparison to the equal time allocation, on-off transmission scheme and half-duplex system. Equal time allocation, denoted by ETA, aims to allocate equal time to all the users, that is, ., $\tau_{i}=T / N$ at any feasible power level $P_{i} \leq P_{\max }$. On-off transmission scheme, denoted by 

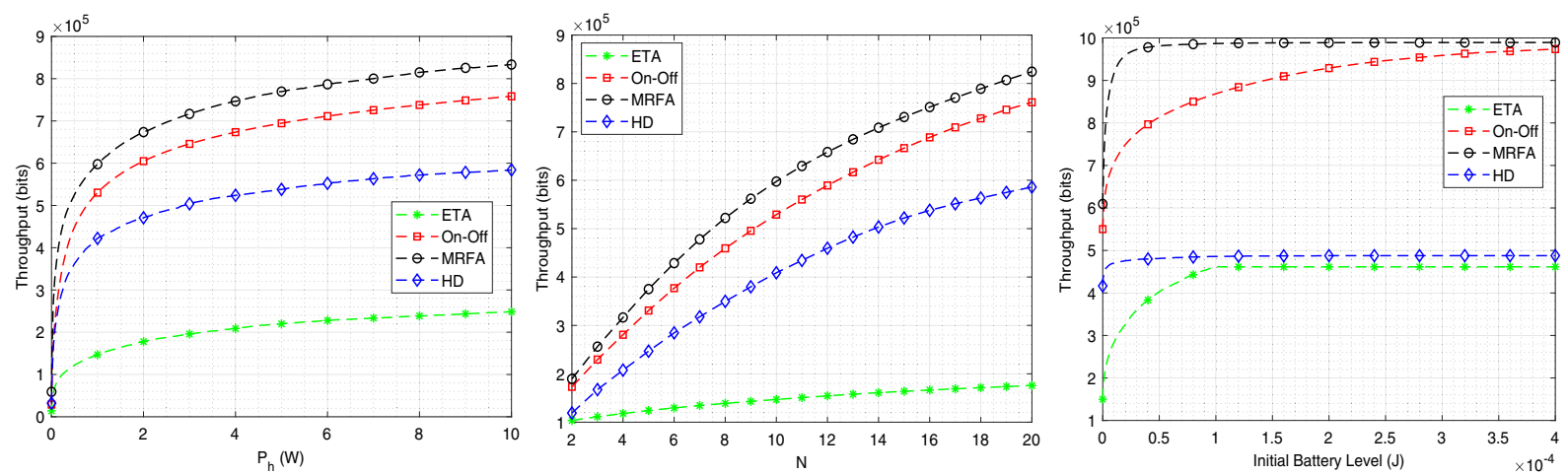

F I G U R E 2 Sum throughput analysis for different HAP transmit powers, network densities and initial battery levels

On-Off, considers the scenario in which the users either transmit at the highest feasible rate from the set $\mathbf{R}$ by using maximum power $P_{\max }$ or remain silent if they can not afford transmission on this power level. ${ }^{13}$ The half-duplex model, denoted by HD, determines the optimal energy harvesting duration and user transmission times with the objective of maximizing the sum throughput of the system for a half duplex model by using the convex optimization technique. ${ }^{15}$ For a fair comparison we incorporate the discrete rates which are achieved by scaling down the continuous value to the nearest discrete rate level, initial battery levels and $P_{\max }$ constraint in the simulations. Simulation results are obtained by averaging over 1000 independent random network realizations and the users are uniformly distributed within a circle of radius $10 \mathrm{~m}$. The attenuation of the links considering large-scale statistics are determined by using the path loss model given by: $P L(d)=P L\left(d_{0}\right)+10 \alpha \log _{10}\left(d / d_{0}\right)+Z$, where $P L(d)$ is the path loss at distance $d$ in $d B, d_{0}$ is the reference distance, $\alpha$ is the path loss exponent, and $Z$ is a zero mean Gaussian random variable with SD $\sigma$. The small-scale fading has been modeled by using Rayleigh fading with scale parameter $\Omega_{i}$ set to mean power level obtained from the large-scale path loss model. The parameters used in the simulations are $\eta_{i}=1$ for $i \in\{1,2, \cdots, N\} ; W=1 \mathrm{MHz} ; d_{0}=1 \mathrm{~m} ; P L\left(d_{0}\right)=30 \mathrm{~dB} ; \alpha=4, \sigma=3$. The self interference coefficient $\beta$ is taken as $-70 \mathrm{dBm}^{14}$ and initial battery levels are assumed to be $10^{-9} \mathrm{~J}$. We use $M=8$ discrete rate with the highest rate level of $1 \mathrm{Mbps}$.

Figure 2 shows the sum throughput for different values of HAP power $P_{h}$, network size $N$ and initial battery level $B$. The sum throughput increases as the $P_{h}$ value increases since energy harvesting rate is directly proportional to the HAP power, hence, user can afford transmission at the higher rate. The proposed MRFA algorithm significantly outperforms the ETA, HD and on-off transmission schemes due to the scheduling and incorporation of discrete rate levels into the solution. The performance improvement of the MRFA algorithm compared to the benchmark schemes increases significantly as the number of users increases, due to the increase in the probability of a user affording to transmit at the highest possible rate. As ETA allocates equal time to all the users, the sum throughput increases almost linearly. The throughput of the HD system is smaller than MRFA because the energy transmission and uplink information transmission cannot happen simultaneously, leading to the under-utilization of the resources. Furthermore, the increase in initial battery level improves the performance since the higher initial battery level allows the users to transmit at higher transmission rate for longer time, which results in a higher sum throughput. When the initial battery level of all the users is high enough so that the whole frame can be occupied at the highest feasible transmission rate, the sum throughput saturates and any further increase in the initial battery level results in no gain and MRFA performs same as On-Off while outperforming ETA and HD. The throughput of HD and ETA is smaller than MRFA because these algorithm do not incorporate scheduling and allocates time to all the users.

\section{7 | CONCLUSION AND FUTURE WORK}

In this paper, we investigate the sum throughput maximization problem considering discrete rate transmission model in a full-duplex wireless powered communication network. We characterize an optimization framework to determine the time allocation, rate adaptation and scheduling subject to maximum transmit power and energy causality of the users. First, we mathematically formulate the problem as a mixed integer nonlinear programming problem, which is difficult to solve for the global optimum in polynomial time. Upon detailed investigation of the problem, we propose a polynomial time complexity heuristic algorithm, which significantly outperforms the equal time allocation, half-duplex and on-off transmission schemes for different network densities, initial battery levels of the users and the hybrid access point transmit powers. For future work, we plan to extend this work by incorporating multiple hybrid access points, formulating and solving the problem using Qubo optimization techniques.

\section{ORCID}




\section{REFERENCES}

1. Liu L, Zhang R, Chua K. Multi-antenna wireless powered communication with energy beamforming. IEEE Trans Commun. 2014;62(12):4349-4361.

2. Di X, Xiong K, Fan P, Yang H, Letaief KB. Optimal resource allocation in wireless powered communication networks with user cooperation. IEEE Trans Wireless Commun. 2017;16(12):7936-7949.

3. $\mathrm{Ju} \mathrm{H}$, Zhang $\mathrm{R}$. Throughput maximization in wireless powered communication networks. IEEE Trans Wireless Commun. 2014;13(1):418-428.

4. Liu G, Yu FR, Ji H, Leung VCM, Li X. In-band full-duplex relaying: a survey, research issues and challenges. IEEE Commun Surv Tutor. 2015;17(2):500-524.

5. Bharadia D, McMilin E, Katti S. Full duplex radios. ACM SIGCOMM. 2013;43:375-386.

6. Kang X, Ho CK, Sun S. Full-duplex wireless-powered communication network with energy causality. IEEE Trans Wireless Commun. 2015;14(10):5539-5551.

7. Ju H, Chang K, Lee M. In-band full-duplex wireless powered communication networks. 2015 17th International Conference on Advanced Communications Technology (ICACT); Seoul, South Korea, 2015: 23-27.

8. Hu J, Xue Y, Yu Q, Yang K. A joint time allocation and UE scheduling algorithm for full-duplex wireless powered communication networks. 2017 IEEE 86th Vehicular Technology Conference (VTC-Fall); Toronto, ON, Canada, 2017: 1-5.

9. Pathak K, Kalamkar SS, Banerjee A. Optimal user scheduling in energy harvesting wireless networks. IEEE Trans Commun. 2018;66(10):4622-4636.

10. Iqbal MS, Sadi Y, Coleri S. Throughput maximization for full duplex wireless powered communication networks. IEEE International Conference on Communications (ICC); Dublin, Ireland, 2020: 1-1.

11. Yadav A, Dobre OA, Ansari N. Energy and traffic aware full-duplex communications for 5G systems. IEEE Access. 2017;5:11278-11290.

12. Iqbal MS, Sadi Y, Coleri S. Minimum length scheduling for full duplex time-critical wireless powered communication networks. IEEE Transactions on Wireless Communications; 2020: 1-1.

13. Iqbal MS, Sadi Y, Coleri S. Optimal on-off transmission policy for full duplex wireless powered communication networks. IEEE International Black Sea Conference on Communications and Networking; Odessa, Ukraine, 2020: 1-1.

14. Iqbal MS, Sadi Y, Ergen SC. Minimum length scheduling for discrete rate based full duplex wireless powered communication networks. International Conference on Ad-Hoc Networks and Wireless; Luxembourg, Luxembourg, 2019: 343-354.

15. Ju H, Zhang R. Throughput maximization in wireless powered communication networks. IEEE Transactions on Wireless Communications. 2014;13(1):418-428. http://dx.doi.org/10.1109/twc.2013.112513.130760.

How to cite this article: Iqbal MS, Sadi Y, Coleri S. Throughput maximization in discrete rate based full duplex wireless powered communication networks. Internet Technology Letters. 2020;e206. https://doi.org/10.1002/itl2.206 